\title{
Field Quality Issues for RHIC Helical Dipole Magnets
}

\author{
M. J. Syphers
}

November 30, 1995

\begin{abstract}
Helical dipole magnets are to be used in RHIC to perform spin manipulations of polarized proton beams. The issue of field quality in these devices leads one to consider the effects of "rotating" multipole fields on beam performance, such as tune shifts and chromaticity. After a short discussion of the intrinsic field nonlinearities of a "twisting" dipole field, estimates of the magnitudes of tune shifts in the RHIC helical Snakes due to magnet harmonics are presented.
\end{abstract}

\section{Helical Dipoles in RHIC}

Helical dipole magnets will be used to maintain polarization in RHIC. These magnets have a dipole cross section, whose field direction rotates about the longitudinal axis of the magnet through $360^{\circ}$ over the length of the device. To make a "Siberian Snake," four superconducting helical dipole magnets are placed in a common cryostat in a straight section in RHIC. (This straight section lies in a "dispersion suppressor region" of the RHIC lattice, roughly the length of a standard RHIC dipole magnet.) The two "inner" Snake magnets have equal and opposite field strengths, as do the two "outer" magnets. The field strengths are chosen so that the overall trajectory of the particles is left undisturbed outside the Snake, but the spin direction of the particles is rotated by $180^{\circ}$ about a horizontal axis pointed $45^{\circ}$ from the longitudinal direction of motion.

In addition, helical dipoles are also used to rotate the spin by $90^{\circ}$ into the longitudinal direction at the interaction points (IP), and back to vertical on the other side of the IP. These Rotators consist again of four helical magnets, but the helicity of two of the magnet coils (second and fourth) are left-handed, whereas the helicity of the other two are right-handed (as are all the Snake magnets). 


\subsection{The helical dipole field}

The field generated by a dipole magnetic moment spiraling around the longitudinal axis ${ }^{1}$ can be found by determining the scalar potential satisfying Laplace's Equation,

$$
\frac{\partial^{2} \Phi}{\partial r^{2}}+\frac{1}{r} \frac{\partial \Phi}{\partial r}+\frac{1}{r^{2}} \frac{\partial^{2} \Phi}{\partial \phi^{2}}+\frac{\partial^{2} \Phi}{\partial z^{2}}=0 .
$$

Using the usual separation of variables, $\Phi=R(r) Q(\phi) Z(z)$, we get three differential equations

$$
\begin{aligned}
\frac{d^{2} R}{d r^{2}}+\frac{1}{r} \frac{d R}{d r}-\left(k^{2}+\frac{\nu^{2}}{r^{2}}\right) R & =0, \\
\frac{d^{2} Q}{d \phi^{2}}+\nu^{2} Q & =0, \\
\frac{d^{2} Z}{d z^{2}}+k^{2} Z & =0 .
\end{aligned}
$$

The repeat period of the helical field is $\lambda=2 \pi /|k|$ and $\nu$ is set equal to one since the central dipole field repeats after $\phi=2 \pi$. We see that the radial solutions are Modified Bessel functions. With these boundary conditions, the fields found by taking $\vec{B}=-\nabla \Phi$ are

$$
\begin{aligned}
B_{r} & =2 B_{0}\left[I_{0}(k r)-\frac{I_{1}(k r)}{k r}\right](\cos k z \sin \phi-\sin k z \cos \phi) \\
B_{\phi} & =2 B_{0} \frac{I_{1}(k r)}{k r}(\cos k z \cos \phi+\sin k z \sin \phi) \\
B_{z} & =-2 B_{0} I_{1}(k r)(\cos k z \cos \phi+\sin k z \sin \phi)
\end{aligned}
$$

In these solutions, we have assumed that the central dipole field $B_{0}$ points vertically upward at the entrance of the helix (i.e., at $z=0$.) If we look at $B_{x}=B_{r} \cos \phi-$ $B_{\phi} \sin \phi, B_{y}=B_{r} \sin \phi+B_{\phi} \cos \phi$ and expand these fields near the longitudinal axis $(x, y$ small $)$, then,

$$
\begin{aligned}
& B_{x} \approx-B_{0}\left\{\left[1+\frac{k^{2}}{8}\left(3 x^{2}+y^{2}\right)\right] \sin k z-\frac{k^{2}}{4} x y \cos k z\right\} \\
& B_{y} \approx B_{0}\left\{\left[1+\frac{k^{2}}{8}\left(x^{2}+3 y^{2}\right)\right] \cos k z-\frac{k^{2}}{4} x y \sin k z\right\} \\
& B_{z} \approx-B_{0} k\left\{1+\frac{k^{2}}{8}\left(x^{2}+y^{2}\right)\right\}[x \cos k z+y \sin k z]
\end{aligned}
$$

To first order, the transverse fields are just $B_{y}=B_{0} \cos k z, B_{x}=-B_{0} \sin k z$ as desired. However, we see that for significant displacements, there are nonlinear terms which will contribute to the particle motion. As we shall see in the next section, the trajectories of particles through the helical magnet systems can be significant.

\footnotetext{
${ }^{1}$ See, for example, J. P. Blewett and R. Chasman, J. App. Phys., 48 (1977) 2692; another derivation can be found from A. Luccio, AGS/AD/Tech. Note No. 399 (1994).
} 


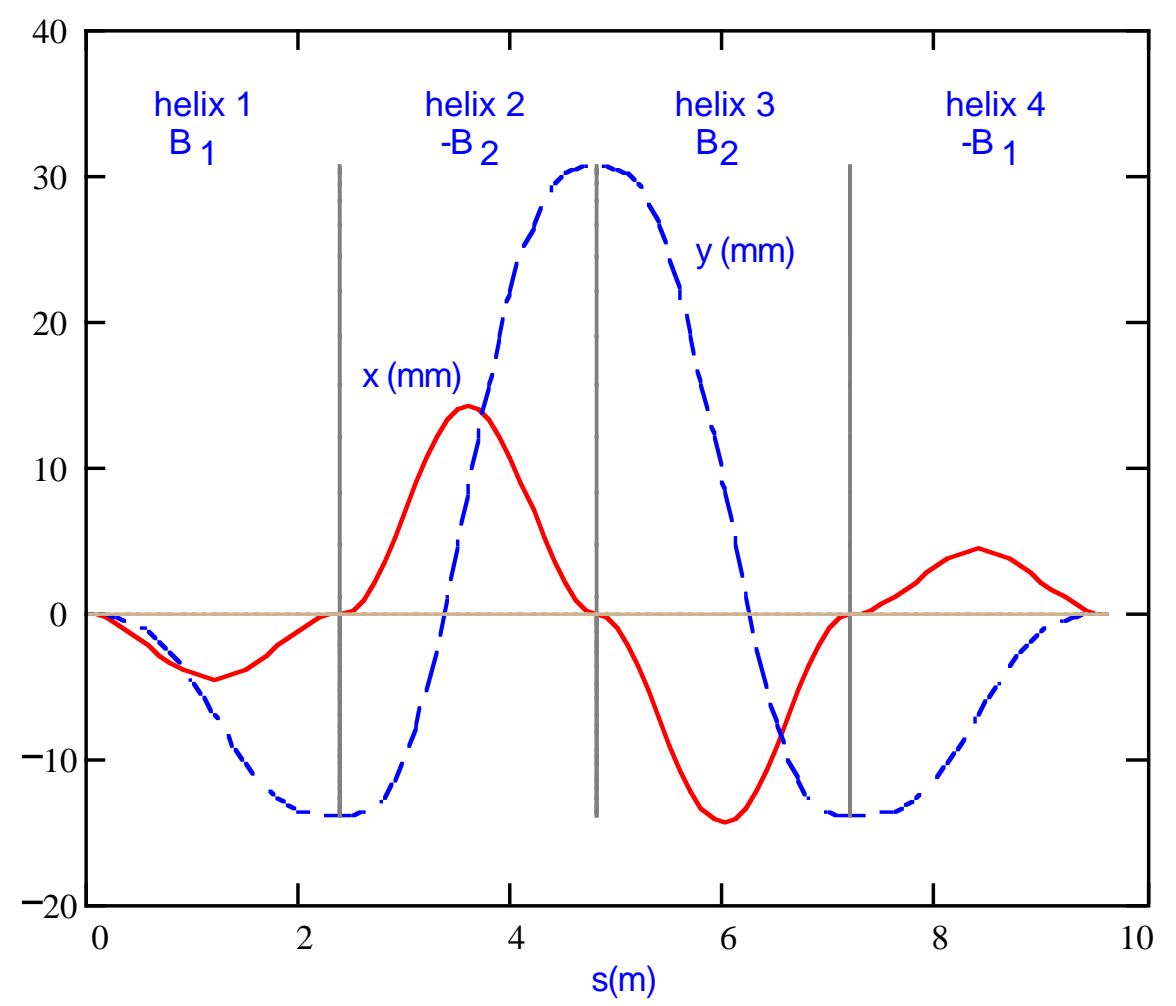

Figure 1: Horizontal and vertical trajectories through a RHIC Snake.

\subsection{Trajectory in a helical dipole field}

For small transverse displacements, the particle motion in a helical field is determined by

$$
\begin{aligned}
& x^{\prime \prime} \approx-\frac{B_{0}}{(B \rho)} \cos k s \\
& y^{\prime \prime} \approx-\frac{B_{0}}{(B \rho)} \sin k s
\end{aligned}
$$

which have solutions

$$
\begin{aligned}
& x(s)=x_{0}-\frac{B_{0}}{(B \rho)} \frac{1}{k^{2}}(1-\cos k s)+x_{0}^{\prime} s \\
& y(s)=y_{0}+\frac{B_{0}}{(B \rho)} \frac{1}{k^{2}} \sin k s+\left(y_{0}^{\prime}-\frac{B_{0}}{(B \rho)} \frac{1}{k}\right) s
\end{aligned}
$$

(Note that here we have switched to the symbol " $s$ " for the longitudinal coordinate.) If the initial trajectory into the helix has zero slope, then we see that after passing through a complete helix (i.e., through a distance $L=\lambda$ ) the trajectory ends 
up again with zero slope, the horizontal displacment is unchanged, and the vertical displacement is altered by an amount

$$
\Delta y=-\frac{B_{0} L}{k(B \rho)} .
$$

Again, this is only approximately true, because we have ignored the nonlinear terms of the fields. But for estimating the magnitudes of effects, this description of the trajectory is adequate.

With the solution above, one can estimate the total vertical deflection within a Snake. For the RHIC system, which has four helical magnets with fields $B_{1},-B_{2}$, $B_{2},-B_{1}$, the maximum deflection will be $-(L / k(B \rho))\left(B_{1}-B_{2}\right)=-\left[(2.4 \mathrm{~m})^{2} /(2 \pi\right.$. $80 \mathrm{~T}-\mathrm{m})](1.2 \mathrm{~T}-4 \mathrm{~T})=32 \mathrm{~mm}$ at the injection energy of $24.4 \mathrm{GeV}$.

The solutions $x(s)$ and $y(s)$ throughout a complete RHIC Snake system (4 helical dipoles) are displayed in Fig. 1; a three dimensional plot of the trajectory is shown in Fig. 2.

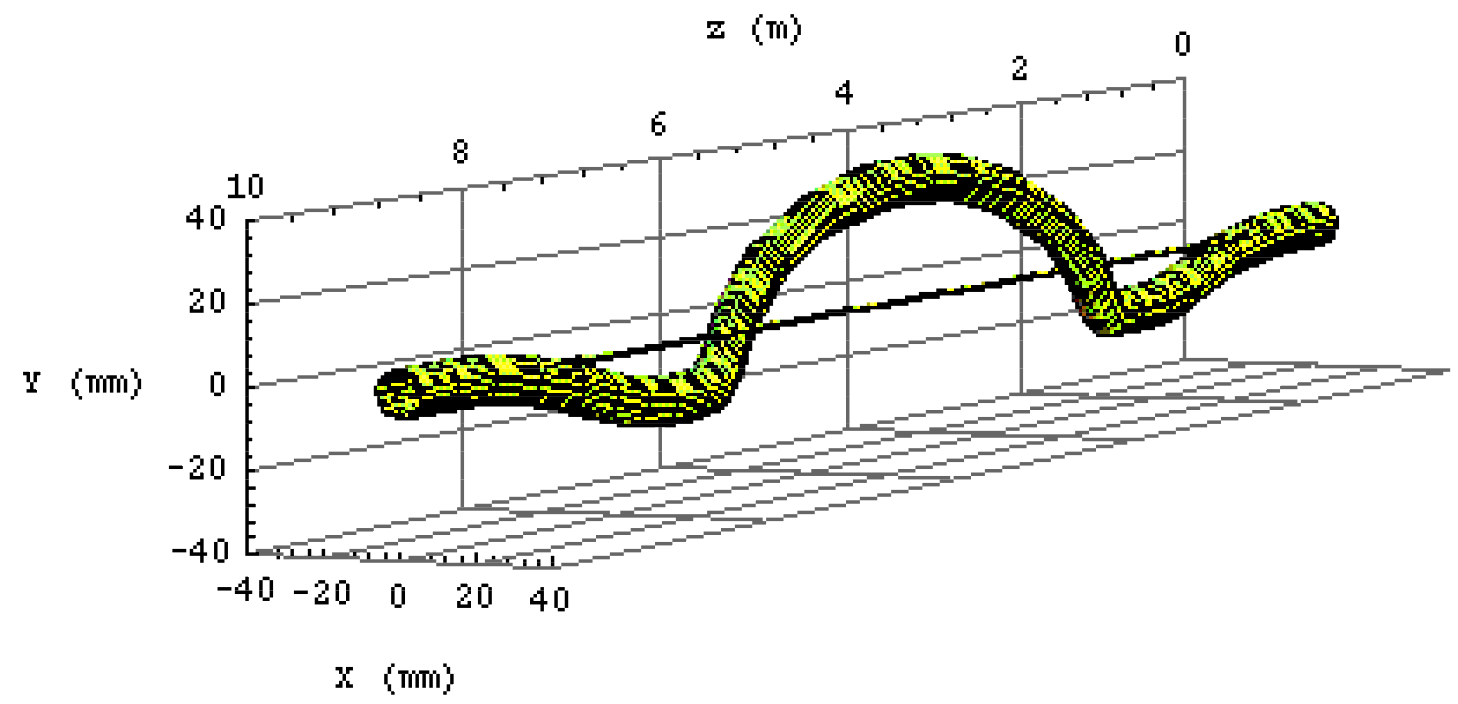

Figure 2: Three dimensional view of the trajectory through a RHIC Snake.

\section{Effects of the Nonlinear Field}

Since the helical twist produces a sextupole-like term (i.e., varies with $x^{2}$ ), this term has the potential to contribute to the chromaticity of RHIC. In addition, the particles do not pass through these devices "on axis." In fact, as we have seen, the trajectories of the particles will pass as much as $30 \mathrm{~mm}$ away from the axis of the snake and rotator magnets. Thus, there is the possibility of feed-down effects from these fields. A tune shift would be generated from the off-center orbit through the sextupole field. The magnitudes of these effects are discussed below. 


\subsection{Chromaticity and Tune Shift from Ideal Snake}

The chromaticity due to a sextupole-like field is given by

$$
\Delta \xi=\frac{1}{4 \pi} \int \frac{\beta(s)}{(B \rho)} D(s) \frac{\partial^{2} B_{y}}{\partial x^{2}} d s
$$

where $\beta(s)$ and $D(s)$ are the amplitude function and dispersion function of the accelerator at the location of the field. For the intrinsic nonlinear field of the helical dipole, $\partial^{2} B_{y} / \partial x^{2}=B_{0}\left(k^{2} / 4\right) \cos k s$ and hence the chromaticity will be given by

$$
\Delta \xi=\frac{k^{2} B_{0}}{16 \pi(B \rho)} \int_{0}^{\lambda} \beta(s) D(s) \cos k s d s
$$

which, for small variations in $\beta$ and $D$ through the helix, will tend toward zero. A more detailed calculation, using the amplitude function and dispersion function variation throughout the Snake, shows that the chromaticity contribution is 0.001 .

The tune shift which is fed down from the quadratic field variation is given by

$$
\begin{aligned}
\Delta \nu & =\frac{1}{4 \pi} \int \frac{\beta(s)}{(B \rho)} \frac{\partial B_{y}}{\partial x} d s \\
& =\frac{k^{2} B_{0}}{16 \pi(B \rho)} \int_{0}^{\lambda} \beta(s)\left[x_{c o}(s) \cos k s-y_{c o}(s) \sin k s\right] d s \\
& \approx-\frac{\beta_{\text {ave }}}{8 k}\left(\frac{B_{0}}{(B \rho)}\right)^{2}
\end{aligned}
$$

for a single helical dipole. For a complete Snake, with four helices, the total tune shift is

$$
\Delta \nu \approx-\frac{\beta_{\text {ave }}}{4 k}\left(\frac{B_{1}^{2}+B_{2}^{2}}{(B \rho)^{2}}\right) .
$$

We see that the tune shift contributions add up, since they depend on the square of the field strengths. For an average $\beta$ of $30 \mathrm{~m}$, a helical dipole length of $2.4 \mathrm{~m}$, and fields of $4 \mathrm{~T}$ and $1.2 \mathrm{~T}$, the tune shift at injection energy $(24 \mathrm{GeV})$ would be rougly -0.007 , or -0.015 for two Snakes. This shift can easily be controlled by the RHIC quadrupole correction system.

It is also interesting to note that the tune shift depends upon the sign of $k$. Thus, in a Rotator, where the magnets are left-handed then right-handed, these tune shift contributions will tend to cancel.

\section{Description of Magnet Error Fields}

Suppose the transverse magnetic field within a thin cross-section of a superconducting helical dipole magnet is described by the usual multipole expansion,

$$
\Delta B_{y h}=B_{0} \operatorname{Re}\left[\sum_{n}\left(b_{n}+\mathrm{i} a_{n}\right)\left(x_{h}+\mathrm{i} y_{h}\right)^{n}\right]
$$




$$
\Delta B_{x h}=B_{0} \operatorname{Im}\left[\sum_{n}\left(b_{n}+\mathrm{i} a_{n}\right)\left(x_{h}+\mathrm{i} y_{h}\right)^{n}\right]
$$

where $b_{n}$ and $a_{n}$ are the "normal" and "skew" components of the field, and $B_{0}$ is the desired dipole field. Here, $\Delta B_{y h}$ and $\Delta B_{x h}$ represent "errors" to the desired field of the helical magnet. The coefficients $b_{n}$ and $a_{n}$ are the quantities which the magnet designers try to control when designing the ideal cross section of the magnet.

Through the helical magnet, this field distribution is rotated by an angle $\theta(s)=$ $2 \pi s / \lambda \equiv k s$ following the distance $s$ along the longitudinal axis of the magnet, where $\lambda$ is the length corresponding to a full twist of the helical field. For the Snake and Rotator magnets in RHIC, $\lambda$ is essentially the magnetic length of the magnet. This rotation of the central field is shown schematically in Fig. 3. Again, $x_{h}, y_{h}$ are the coordinates corresponding to the central field (i.e., the central dipole field points along $y_{h}$ ), while $x$ and $y$ are the "real space" coordinates. Then, since $\Delta B_{x}=$

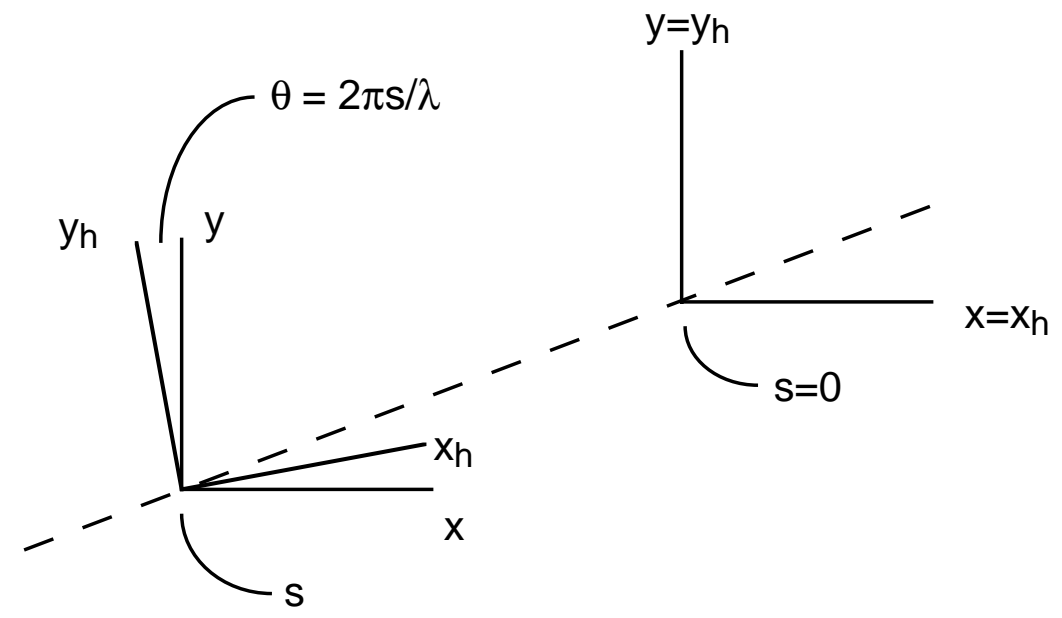

Figure 3: Rotating coordinates of dipole field components.

$\Delta B_{x h} \cos k s-\Delta B_{y h} \sin k s$, and $\Delta B_{y}=\Delta B_{x h} \sin k s+\Delta B_{y h} \cos k s$ we can express the field error at $x$ and $y$ in terms of the usual multipole coefficients:

$$
\begin{aligned}
\Delta B_{y}= & B_{0} b_{n} r^{n}[\cos n \phi \cos (n+1) k s+\sin n \phi \sin (n+1) k s] \\
& -B_{0} a_{n} r^{n}[\sin n \phi \cos (n+1) k s-\cos n \phi \sin (n+1) k s] \\
\Delta B_{x}= & B_{0} b_{n} r^{n}[\sin n \phi \cos (n+1) k s-\cos n \phi \sin (n+1) k s] \\
& +B_{0} a_{n} r^{n}[\cos n \phi \cos (n+1) k s+\sin n \phi \sin (n+1) k s]
\end{aligned}
$$

where, as usual, $x=r \cos \phi$ and $y=r \sin \phi$. For simplicity, we will assume the skew components are zero in the subsequent discussion, and we will examine the effects of the "allowed" normal multipole terms, which will be the most significant. 


\subsection{Dipole Error Field}

For a dipole error field, $n=0$, the above expressions reduce to

$$
\begin{aligned}
\Delta B_{y} & =B_{0} b_{0} \cos k s \\
\Delta B_{x} & =B_{0} b_{0} \sin k s
\end{aligned}
$$

\subsection{Sextupole Error Field}

For a sextupole error field, $n=2$, and

$$
\begin{aligned}
\Delta B_{y} & =B_{0} b_{2}\left[\left(x^{2}-y^{2}\right) \cos 3 k s+2 x y \sin 3 k s\right] \\
\Delta B_{x} & =B_{0} b_{2}\left[2 x y \cos 3 k s-\left(x^{2}-y^{2}\right) \sin 3 k s\right]
\end{aligned}
$$

\subsection{Decapole Error Field}

For a decapole error field, $n=4$, and

$$
\begin{aligned}
\Delta B_{y} & =B_{0} b_{4}\left[\left(x^{4}-6 x^{2} y^{2}+y^{4}\right) \cos 5 k s+4\left(x^{3} y-x y^{3}\right) \sin 5 k s\right] \\
\Delta B_{x} & =B_{0} b_{4}\left[4\left(x^{3} y-x y^{3}\right) \cos 5 k s-\left(x^{4}-6 x^{2} y^{2}+y^{4}\right) \sin 5 k s\right]
\end{aligned}
$$

\section{Effects of Multipole Errors}

As before, we would expect multipole errors to generate chromaticity and tune shifts. One could argue that because the helical fields rotate through $360^{\circ}$ for each helix, that the effects of the nonlinear fields would self cancel. This is roughly true for the chromaticity. However, again because of the large orbit excursions, feed down effects need to be considered.

The allowed multipole coefficients for one magnet design are shown in Table 1. The multipoles are in units of $10^{-4}$ of $B_{0}$ at $3.1 \mathrm{~cm}$ radius. The use of a $14 \mathrm{in}$. versus

\begin{tabular}{|l|c|c|}
\hline Design & $b_{2}$ & $b_{4}$ \\
\hline 14 in. Yoke & & \\
1 current & -55 & -7.2 \\
2 currents & 0.5 & 1 \\
\hline 16 in. Yoke & & \\
1 current & 2 & -3.4 \\
2 currents & 0.5 & 0.5 \\
\hline
\end{tabular}

Table 1: Multipole coefficients for helical magnet design. 
a 16 in. iron yoke will influence the size of the allowed multipoles, as the iron will begin to saturate with the smaller yoke. This saturation can be partially compensated through the use of separate currents in the inner and outer layers of the magnet coil. The question of whether 55 units of $b_{2}$ and roughly 10 units of $b_{4}$ are tolerable are addressed below.

\subsection{Chromaticity Contribution}

As before, the chromaticity due to the $b_{2}$ field can be estimated. Here,

$$
\Delta \xi=\frac{b_{2}}{2 \pi(B \rho)} \int_{0}^{4 \lambda} B_{0}(s) \beta(s) D(s) \cos 3 k s d s
$$

which will be roughly zero, assuming the lattice functions do not vary much through each magnet. A detailed calculation gives a result of $\Delta \xi$ of approximately $10^{-4}$.

\subsection{Tune shift due to $b_{2}$ multipole error}

Due to the particle trajectory through the Snake, the tune shift generated by a $b_{2}$ coefficient in the magnet design will be

$$
\Delta \nu=\frac{b_{2}}{2 \pi} \int_{0}^{4 \lambda} \beta(s) \frac{B_{0}(s)}{(B \rho)}\left[x_{c o}(s) \cos 3 k s+y_{c o}(s) \sin 3 k s\right] d s
$$

Since the integral is taken over all four magnets in the Snake, the central field $B_{0}(s)$ is written as a function of $s$. Carrying out the calculation as before, the tune shift due to the $b_{2}$ coefficient in the four magnets becomes

$$
\begin{aligned}
\Delta \nu & =\frac{b_{2}}{2 \pi} \frac{\beta_{\text {ave }}}{(B \rho)} \sum_{i=1}^{4} B_{i} \cdot\left(-\frac{6 \pi}{9 k^{3}} \frac{B_{i}}{(B \rho)}\right) \\
& =-\frac{2 b_{2} \beta_{\text {ave }}}{3 k^{3}} \frac{\left[B_{1}^{2}+B_{2}^{2}\right]}{(B \rho)^{2}}
\end{aligned}
$$

where $B_{1}$ and $B_{2}$ are the values of the central dipole fields of the outer and inner helical magnets. Also, this expression assumes the same value of $b_{2}$ in the four magnets, which may actually depend upon the magnetic field strength.

To estimate the size of the effect, we again use the parameters $\beta_{\text {ave }}=30 \mathrm{~m}, \lambda=$ $2.4 \mathrm{~m}$, fields of $4 \mathrm{~T}$ and $1.2 \mathrm{~T}$, and we'll use the large value for $b_{2}$ given in Table 1 , namely $b_{2}=-55 \times 10^{-4} /(3.1 \mathrm{~cm})^{2}$. This yields a tune shift of $\Delta \nu=-0.017$ for one Snake, or a total shift of -0.034 for two Snakes in one ring. Again, this is a noticeable tune shift, but one that can be compensated easily by the RHIC correction system. 


\subsection{Tune Spread due to $b_{4}$ Feed Down}

Lastly, we estimate the tune spread induced by feed down of a $b_{4}$ multipole coefficient in the helical magnet design. Due to an octupole-like magnetic field $\left(\sim x^{3}\right)$, the tune of a particle will vary quadratically with betatron amplitude according to:

$$
\Delta \nu(a)=\frac{1}{2 \pi} \frac{3}{8} K a^{2}
$$

where

$$
K \equiv \frac{\beta_{0}}{6(B \rho)} \int\left(\frac{\beta}{\beta_{0}}\right)^{2} \frac{\partial^{3} B_{y}}{\partial x^{3}} d s .
$$

Here, $\beta_{0}$ is the value of the amplitude function where the particle's oscillation amplitude $a$ is measured. So, a field error with a $b_{4}$ contribution

$$
\Delta B_{y}=B_{0} b_{4}\left[\left(x^{4}-6 x^{2} y^{2}+y^{4}\right) \cos 5 k s+4\left(x^{3} y-x y^{3}\right) \sin 5 k s\right]
$$

will generate a non-zero feed down into an octupole-like field which yields

$$
\frac{\partial^{3} \Delta B_{y}}{\partial x^{3}}=B_{0} b_{4}[24 x \cos 5 k s+24 y \sin 5 k s] .
$$

Integrating over a single helical magnet, and again assuming the amplitude function does not vary much over this region, the contribution to the tune shift will be

$$
\begin{aligned}
\Delta \nu(a) & \approx \frac{1}{2 \pi} \frac{3}{8} \frac{24}{6} \frac{B_{0} b_{4}}{(B \rho)} \frac{a^{2}}{\beta_{0}} \beta_{\text {ave }}^{2}\left(\frac{2 \pi}{5} \frac{B_{0}}{(B \rho) k^{3}}\right) \\
& =\frac{3}{10} \frac{B_{0}^{2} b_{4}}{(B \rho)^{2}} \frac{a^{2}}{\beta_{0}} \frac{\beta_{\text {ave }}^{2}}{k^{3}}
\end{aligned}
$$

for a single helical magnet. For one complete Snake, the tune shift is thus approximately

$$
\Delta \nu\left(\epsilon_{n}\right) \approx \frac{3}{5 \pi} \frac{\beta_{a v e}^{2} b_{4}}{\gamma k^{3}} \frac{\left[B_{1}^{2}+B_{2}^{2}\right]}{(B \rho)^{2}} \epsilon_{n}
$$

where $\epsilon_{n}$ is the normalized emittance of the single particle, and $\gamma$ is the relativistic Lorentz factor.

For the RHIC beam with a $95 \%$ normalized emittance of $20 \pi \mathrm{mm}-\mathrm{mr}$, there would be a tune spread at injection due to a $b_{4}$ of -7 units on the order of

$$
\begin{aligned}
\delta \nu & \approx \frac{3}{5 \pi} \frac{(30 \mathrm{~m})^{2}\left(-7 \cdot 10^{-4} /(3.1 \mathrm{~cm})^{4}\right)}{26(2 \pi / 2.4 \mathrm{~m})^{3}} \frac{\left[(4 \mathrm{~T})^{2}+(1.2 \mathrm{~T})^{2}\right]}{(80 \mathrm{Tm})^{2}} 20 \pi \times 10^{-6} \mathrm{~m} \\
& =-5 \times 10^{-5},
\end{aligned}
$$

or, $-10^{-4}$ for two Snakes. Even if the emittance were $50 \pi \mathrm{mm}-\mathrm{mr}$, and the decapole coefficient were 20 units, the tune spread across the beam would be less than 0.001 . 
Thus, the effect of $b_{4}$ in the Snake cross section design should not be a concern for RHIC operation so long as it is within reasonable bounds.

It is interesting to note that the next term in the field expansion Eq. 8 will be of order $x^{4}$ and will thus feed down into an octupole-like term. The tune spread due to this intrinsic nonlinear field is roughly 1000 times smaller than that estimated above.

\section{Concluding Remarks}

We see that the effects of the Snakes in RHIC on beam performance should be rather benign, even for seemingly large multipole content in the magnet designs. The tune shifts and chromaticities due to the intrinsic nonlinear fields as well as multipole error fields inevitible in the magnet design and construction appear at first glance to be insignificant in comparison with the compensations that will need to be performed due to the errors in the main dipole and quadrupole magnets of the ring. The fact that the Snake magnets are not tuned during acceleration or storage, means that operationally the compensation of their effects should be straightforward. The fact that the tune shifts considered here vary with odd powers of $k$, tells us that the Rotators should contribute even less to these effects, since their helicity switches every other magnet. We did not look at the effects induced by the skew multipole coefficients, which will lead to coupling of the transverse planes. However, these effects should be much smaller than the coupling induced by the helical field itself (in particular, the rather large $B_{s}$ at large orbit excursions), which has already been estimated to be small. ${ }^{2}$

One could contemplate designing the magnet cross sections with multipole fields which could partially compensate the intrinsic nonlinearities of the helical dipole field. However, since all of these effects are small, this would not be a worthwhile endeavor. The major operational challenge for the Snakes and Rotators will be the proper manipulation of the particle orbit and particle spin, as it should be.

\footnotetext{
${ }^{2}$ E. D. Courant, RHIC/AP/47, November 1994; F. Pilat, RHIC/AP/56, February 1995.
} 